\title{
PICOSECOND TIME-RESOLVED FLUORESCENCE OF PHYCOBILIPROTEINS: SUBUNITS OF PHYCOCYANIN FROM Mastigocladus laminosus
}

\author{
R. FISCHER, J. GOTTSTEIN and H. SCHEER ${ }^{\dagger}$ \\ Botanisches Institut der Universität, Menzinger Str. 67, D-8000 München 19 \\ (F.R.G.) \\ P. GEISELHART and S. SCHNEIDER ${ }^{\dagger}$ \\ Institut für Physikalische und Theoretische Chemie der Technischen \\ Universität München, 8046 Garching (F.R.G.)
}

(Received April 19, 1989; accepted July 13, 1989)

Keywords. Cyanobacteria, photosynthesis, phycobiliproteins, phycocyanin, subunit, aggregation, fluorescence polarization, fluorescence kinetics, energy transfer.

\section{Summary}

The $\alpha$ - and $\beta$-subunits of C-phycocyanin from Mastigocladus laminosus were prepared according to revised procedures. Both subunits are isolated as dimers, which can be dissociated into monomers with detergent mixtures. The fluorescence decay kinetics are similar for the respective monomers and dimers. In no case could they be fitted by only one ( $\alpha$-subunit) or two exponentials ( $\beta$-subunit) which are predicted by theory for samples with a unique chromophore-protein arrangement containing one and two chromophores, respectively. It is suggested that there exists a heterogeneity among the chromophores of the subunits, which may persist in the highly aggregated complexes present in cyanobacterial antennas.

\section{Introduction}

Cyanobacteria and rhodophytes contain special light-harvesting complexes, the phycobilisomes, which are situated at the cytoplasmic surface of the thylakoid membranes [1-6]. They are complex structures containing phycobiliprotein aggregates (phycocyanin (PC), phycoerythrin, phycoerythrocyanin, allophycocyanin) and so-called linker peptides. All phyco-

$\dagger$ Authors to whom reprint requests should be addressed. S. Schneider is presently affiliated with Institut für Physikalische und Theoretische Chemie der Universität, Egerland Str. 3, 8520 Erlangen, F.R.G. 
biliproteins and some of the linkers contain covalently bound bile-pigment chromophores. The phycobilisomes comprise highly ordered arrays of some 400 - 2000 chromophores which collect light and transfer the absorbed energy efficiently to photosystem II.

The phycobilisomes are a unique system in which energy transfer in a photosynthetic antenna can be studied. They can be reversibly dissociated into well-defined fragments [1 - 8]. The crystal structures of several phycobiliproteins have been determined with atomic resolution $[9,10]$, and the primary structures are known for a variety of phycobiliproteins and linker polypeptides $[11,12]$. Much interest has consequently been focused on the spectroscopy and excited-state kinetics of such fragments with the aim to understand the energy transfer mechanism of the entire system [see $1-6$ and $13-17$ for leading references].

Rather unexpectedly, even the most simple of such complexes, e.g. PC $\alpha$ subunits, showed multicomponent decay patterns $[18,19]$. The $\alpha$-subunit is the smallest PC fragment carrying only a single phycocyanobilin chromophore. In time-resolved fluorescence measurements a monoexponential decay is therefore expected for a structurally homogeneous sample. One possible origin for the observed multiexponential decay is a natural microheterogeneity of the biliprotein chromophore sites [20-22]. Another possibility is that this decay pattern is artefactual (i.e. due to the formation of homo-aggregates) and hence of no relevance to the situation in phycobilisomes. Since subunit isolation in C-phycocyanin (C-PC) involves a complete unfolding/separation/refolding sequence [23-25], another possible source for artefacts is that the (under such conditions) labile bile-pigment chromophores [26] or the polypeptides are damaged in the process of separation. In order to understand the origin for the complex decay kinetics, we have reinvestigated the aggregation and fluorescence decay kinetics of both the $\alpha$-and $\beta$-subunits of PC prepared under protective conditions, from Mastigocladus laminosus.

\section{Materials and methods}

Mastigocladus laminosus was cultivated in Castenholz medium [27] in 3001 batches at the Gesellschaft für Biotechnologische Forschung, Stöckheim (F.R.G.), and stored deep frozen.

The preparation of PC followed the method of Füglistaller et al. [24]. The subunits of PC were obtained by an improved isoelectric focusing procedure in $7 \mathrm{M}$ urea. The modifications of the previously used procedure [25] were as follows. The dextran gel was presaturated with argon, and the electrofocusing done at $2{ }^{\circ} \mathrm{C}$ under argon and in darkness. After elution from the gel, the subunits were renatured by dialysis against potassium phosphate buffer $(100 \mathrm{mM}$; pH 7.0) first at room temperature for $15 \mathrm{~h}$ and subsequently at $4{ }^{\circ} \mathrm{C}$ for $6 \mathrm{~h}$. A further purification of the $\beta$-subunit was achieved by two subsequent ammonium sulfate precipitations ( $40 \%$ each). 
For dissociation of the homodimers obtained under these conditions (vide infra), solution with an absorbtion in the red band of about $2.5(\alpha$ subunit) and $1.5 \mathrm{~cm}^{-1}$ ( $\beta$-subunit) were treated with a mixture of the detergents, R-AMDML and T-DF12 (Rewo and Steinau, F.R.G.) (0.2 and 0.5\% each, respectively, for the $\alpha$-and $\beta$-subunits. The native state of the subunits was controlled by circular dichroism and the relative amplitudes of the red and near-UV absorptions, which are very sensitive to chromophore geometry [28 - 30].

The aggregation state of the subunits was studied by sucrose density gradient centrifugation [31] in a model Ultrospin 55 ultracentrifuge (LKB, Munich, F.R.G.) at $238000 \times$ g. Trimeric PC from Mastigocladus laminosus $(106.1 \mathrm{kDa})$, horse-heart myoglobin $(17.5 \mathrm{kDa})$ and cytochrome c $(12.4$ $\mathrm{kDa}$ ) were used as reference. The colored bands were withdrawn and quantitated by absorption spectroscopy.

Absorption spectra were recorded on a Lambda 2 (Perkin-Elmer, Überlingen, F.R.G.) or a model 8451 A spectrophotometer (HewlettPackard, Corvallis, OR, U.S.A.). Fluorescence polarization spectra were determined in a model 221 fluorimeter (Spex, München, F.R.G.).

For picosecond time-resolved fluorescence measurements, the samples were excited at low intensity (about $10^{13}$ photons per pulse) by the pulse train of a mode-locked picosecond dye-laser (pulsewidth less than or equal to $3 \mathrm{ps)}$ at a repetition rate of $82 \mathrm{MHz}$. The fluorescence decay traces were recorded with a synchroscan streak camera (Hamamatsu Photonics, Hamamatsu, Japan) with polarizers parallel $\left(I_{\|}\right)$or orthogonal $\left(I_{\perp}\right)$ to the polarization of the exciting radiation. Alternatively, measurements were made with the analyzer under $54^{\circ}$ (magic angle) to eliminate the effects of chromophore reorientation or energy transfer on the isotropic decay. The monitored fluorescence was spectrally selected by interference filters with a bandwidth of $6-8 \mathrm{~nm}$. The experimental decay curves were analyzed by means of a nonlinear least-squares fit routine on the basis of a multiexponential decay law $[32,33]$.

\section{Results and discussion}

\subsection{Subunit preparation}

The subunit preparation followed essentially the procedure of KöstReyes et al. [25], but involved several additional safeguards. The original procedure generally yielded subunits which on SDS-PAGE showed one or more bands moving ahead of the main colored band. This may arise in part from protein degradation. Since the phycocyanobilin chromophores are very labile when uncoupled from the protein [26], oxidative degradation is a likely cause for this heterogeneity. Degradation was particularly critical for the $\beta$-subunit, which gave colored and uncolored extra bands moving faster than the $\beta$-subunit on SDS-polyacrylamide gels. According to their relative mobilities, and the chromophore:protein ratios (which were determined by staining intensity vs. absorption before staining), these additional bands 
carried one or no chromophore. They are therefore believed to originate from sequential oxidative degradation of the two chromophores in the $\beta$ subunit. Effective protective measures were: (1) to run the electrofocusing gel under argon in a suitably modified apparatus (addition of ascorbate to the gel was less reliable); (2) to maintain low temperature $\left(2{ }^{\circ} \mathrm{C}\right)$, which required a concomitant decrease of the urea concentration to $7 \mathrm{M}$ and a carefully controlled renaturation.

\subsection{Aggregation state of subunits}

When investigated by the sedimetation velocity method [31], both PC subunits proved dimeric. Attempts to dissociate these dimers by chaotropic salts, $\mathrm{KSCN}$ or $\mathrm{LiClO}_{4}$, were unsuccessful. The two PC subunits have very similar three-dimensional structures [9], which may lead to homorather than hetero-aggregation in the absence of the correct partner. In the integral PC, chaotropic salts are also ineffective in dissociating heterodimers ("monomers") into the subunits, but rather dissociate higher aggregates into such heterodimers.

Further attempts to obtain monomeric subunits concentrated on the use of detergents. The ones tested fell into two classes. They were either ineffective, or they were too "strong" and led not only to monomerization, but to (partial or complete) unfolding of the subunits at the same time, as judged from the relative intensities of the two absorption bands (details will be published separately). A more successful approach was to use mixtures of detergents of either class. A mixture of the nonionic T-DF12 and the zwitterionic detergent R-AMDML $(0.2$ and $0.5 \%$ each for the $\alpha$ - and $\beta$-subunits, respectively) dissociated the subunits into monomers. However, even under these optimum conditions the absorption spectra indicated already a partial unfolding (decrease of $Q_{\mathrm{red} / \mathrm{UV}}$, the relative absorptivities in the red and nearUV maxima), which increased upon prolonged standing.

\subsection{Fluorescence polarization}

Additional evidence for aggregation of subunits to homodimers comes from fluorescence anisotropy measurements in the presence and absence of detergents. The fluorescence and polarization spectra (Fig. 1) are similar to those reported for the subunits of this pigment [16]. The polarization spectra of the $\alpha$-subunit are rather independent of the excitation wavelength, a fact which indicates that emission takes place from the excited chromophore only. The polarization spectra of the $\beta$-subunit show a pronounced step. This is expected for the $\beta$-subunit with two or more non-equivalent chromophores coupled by energy transfer. Polarization reaches a maximum value at long. wavelength excitation, which is higher than for the $\alpha$-subunit. In view of the chromophore heterogeneity and aggregation, we have not attempted to evaluate an angle between excitation and emission transition dipole moments from these data.

The polarization spectra do not change significantly when the subunits were treated with increasing amounts of a 1:1 mixture of the two aforemen- 


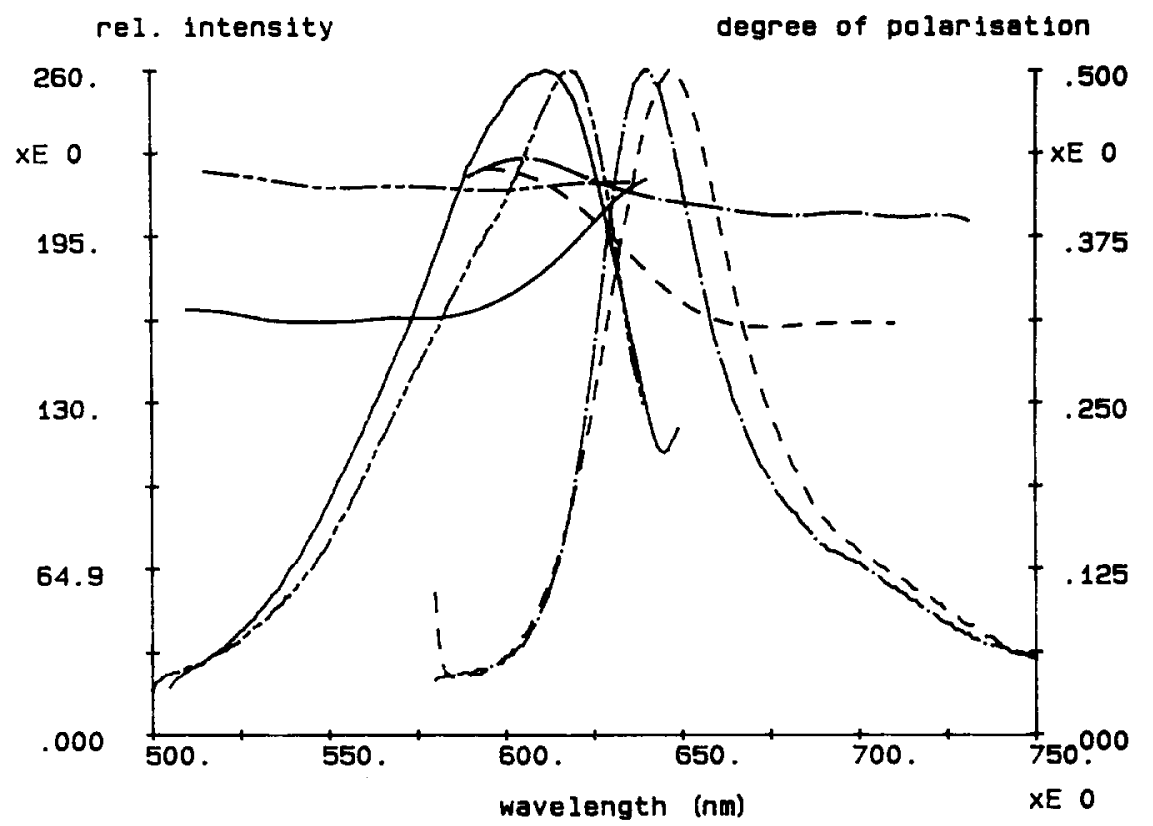

Fig. 1. Fluorescence excitation, emission and polarization spectra of C-PC subunits (homodimer) from $M$. laminosus $\alpha$-subunit: excitation and excitation polarization $(---)\left(\lambda_{\text {det }}=650 \mathrm{~nm}\right)$; emission and emission polarization $(-\cdot-)\left(\lambda_{\text {exc }}=580\right.$ $\mathrm{nm})$. $\beta$-subunit: excitation and excitation polarization $(-)\left(\lambda_{\mathrm{det}}=650 \mathrm{~nm}\right)$; emission and emission polarization $(-\cdots)\left(\lambda_{\text {exc }}=560 \mathrm{~nm}\right)$. The excitation and emission spectra are normalized to equal maximum intensity.

tioned detergents. However, there is an initial increase of the degree of polarization, followed by a plateau at higher detergent concentrations (Fig. 2). The emission polarization of the $\alpha$-subunit reaches 0.36 and that of the $\beta$ subunit, in case of long-wavelength excitation, almost the theoretical maximum of 0.5 . The changes at low detergent concentrations can be due to a decreased rotational relaxation, conformational changes, or a decreased energy transfer. Addition of detergents are likely to increase rotational relaxation. Pronounced conformational changes should also be seen in the absorption spectra. We therefore take the decreased energy transfer as the most likely cause, which is in line with a disaggregation of subunit homoaggregates.

Since addition of increasing amounts of detergents leads at the same time to progressive denaturation of the subunits (decrease of the relative band intensities, $Q_{\text {red/Uv }}$ not shown), there is only a narrow gap at which native monomeric subunits can be obtained. Even under these conditions, the samples tend to degrade slowly and are much more unstable than the homo-dimeric subunits, a fact which imposed restrictions on the measurements with such samples. 
degree of pol.

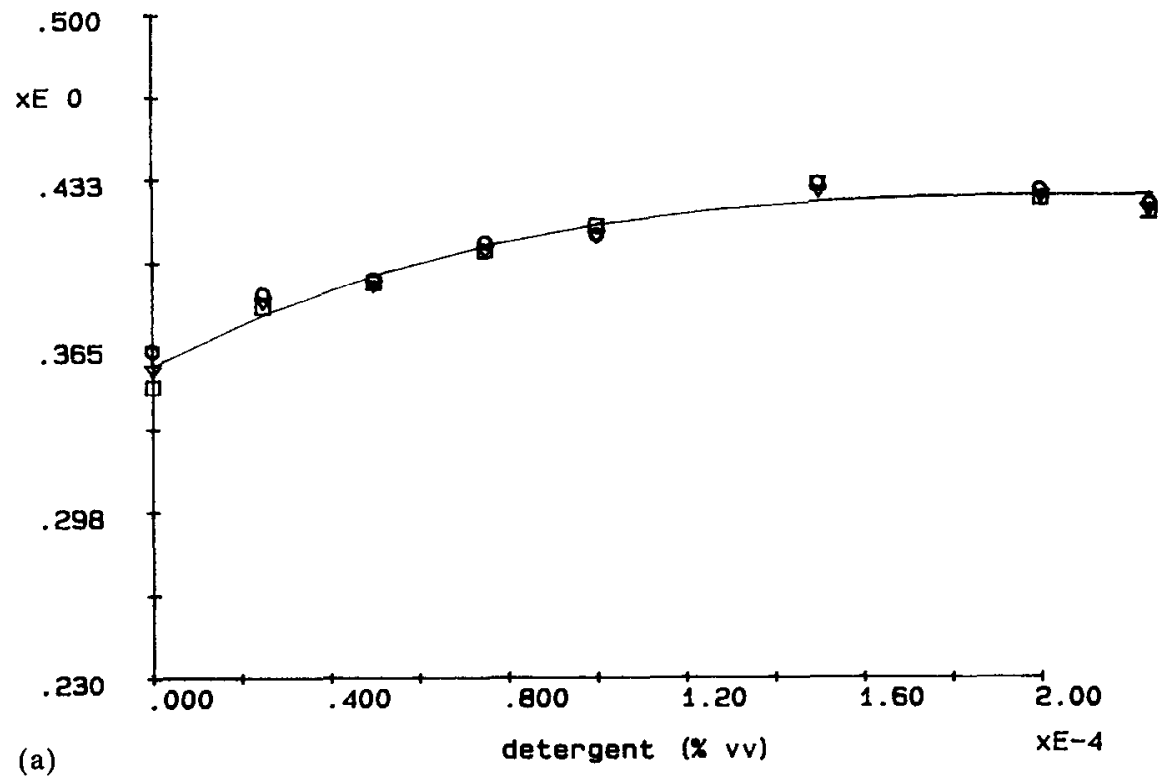

degree of pol.

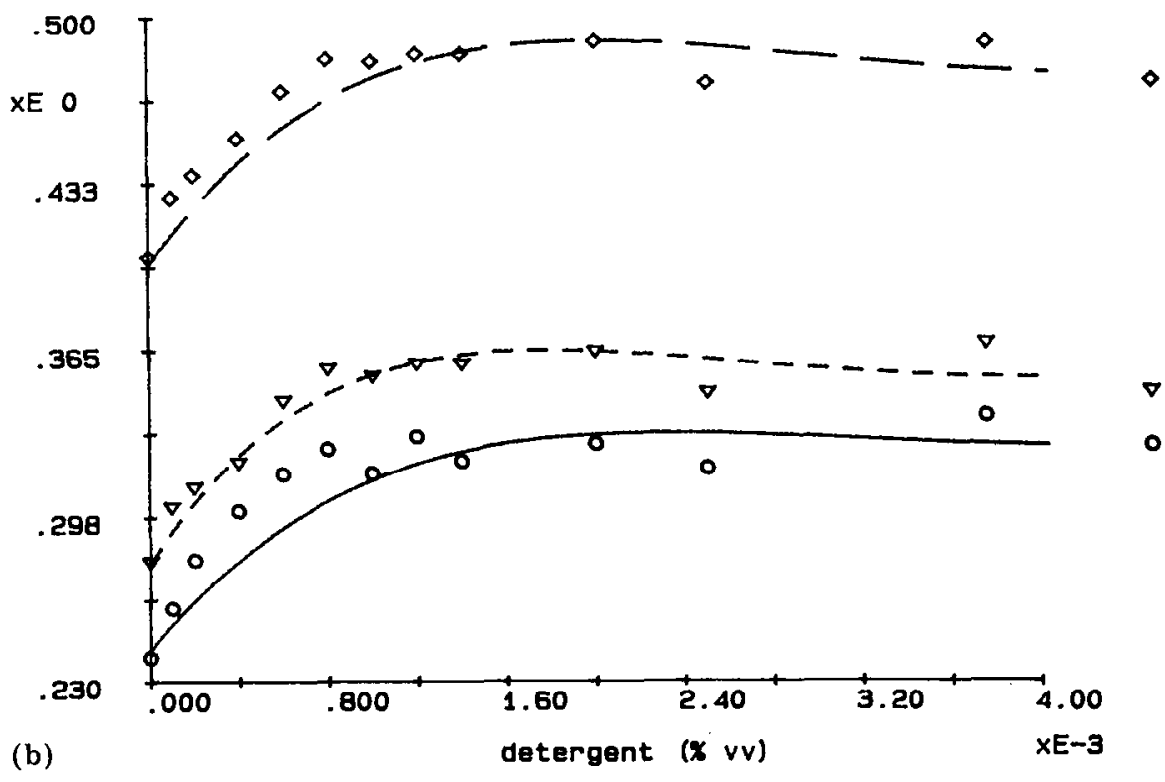

Fig. 2. Effect of detergent concentration on fluorescence polarization of PC subunits (1:1 mixture of a R-AMDML and T-DF12). (a) $\alpha$-subunit: $\lambda_{\text {det }}=645 \mathrm{~nm} ; \lambda_{\text {exc }}=540$ (D), $580(\nabla)$ and $616 \mathrm{~nm}(0)$. (b) $\beta$-subunit: $\lambda_{\text {det }}=650 \mathrm{~nm}, \lambda_{\text {exc }}=598(0), 614(\nabla)$ and $640 \mathrm{~nm}(\diamond)$. 

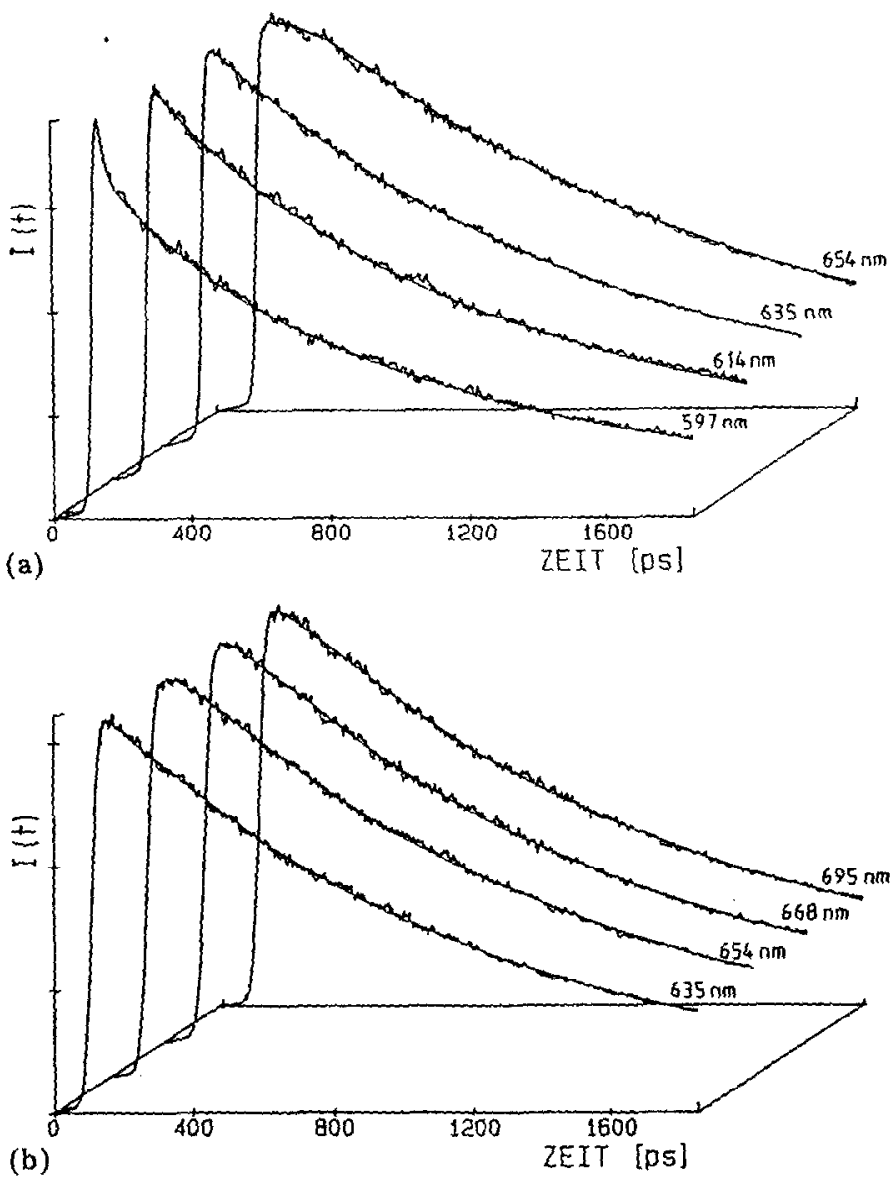

(b)

Fig. 3. Fluorescence decay of $\alpha$-subunit (homodimer) in dependence on the detection wavelength. (a) $\lambda_{\text {exc }}=580 \mathrm{~nm}$; (b) $\lambda_{e x c}=620 \mathrm{~nm}$.

\subsection{Fluorescence decay kinetics: $\alpha$-subunit}

Fluorescence decay measurements were performed for different excitation and detection wavelengths (Fig. 3). The fit parameters, i.e. lifetimes and amplitudes, are summarized in Table 1 . It should be pointed out here that the absolute amplitudes of different measurements are not directly comparable, because of experimental conditions the decay curves are not uniformly normalized.

In all cases, the observed fluorescence decay of the $\alpha$-subunit is not monoexponential. The decay traces are clearly dominated by a long-lived component with a lifetime of 1.1-1.2 ns, but additional components are found. Especially at $580 \mathrm{~nm}$ excitation and $597 \mathrm{~nm}$ detection, a very fast component with only 16 ps lifetime is evident. By additional cut-off filters in detection it was ascertained that there was no scattered excitation light, but only emission from the sample. At long-wavelength excitation $(620 \mathrm{~nm})$, rising fluorescence components (negative amplitudes) with comparable life- 
TABLE 1

Lifetime $(\tau)$ in picoseconds with amplitude (A) in parentheses, for the $\alpha$ - and $\beta$-subunits, at various wavelengths of excitation $\left(\lambda_{\text {exe }}\right)$ and detection $\left(\lambda_{\text {det }}\right)$

\begin{tabular}{|c|c|c|c|}
\hline \multirow{2}{*}{$\begin{array}{l}\lambda_{\text {det }} \\
(\mathrm{nm})\end{array}$} & \multicolumn{3}{|l|}{$\underline{\lambda_{e x c}(\mathrm{~nm})}$} \\
\hline & 580 & 600 & 620 \\
\hline \multicolumn{4}{|c|}{$\alpha-S u b u n i t$} \\
\hline $\begin{array}{l}\alpha-S u b \\
597\end{array}$ & $\begin{array}{c}16(210) \\
180(85) \\
1130(320)\end{array}$ & & \\
\hline \multirow[t]{2}{*}{614} & $160(130)$ & & \\
\hline & $1160(660)$ & & \\
\hline \multirow[t]{2}{*}{635} & $200(220)$ & & - \\
\hline & $1210(1720)$ & & $1120(2790)$ \\
\hline 654 & $95(-100)$ & & $25(-510)$ \\
\hline 668 & $1230(1510)$ & & $\begin{array}{c}1150(3050) \\
15(-1180)\end{array}$ \\
\hline \multirow[t]{2}{*}{695} & & & $\begin{array}{c}1130(3110) \\
10(-1060)\end{array}$ \\
\hline & & & $1240(2840)$ \\
\hline \multicolumn{4}{|c|}{$\beta$-Subunit } \\
\hline 597 & $\begin{array}{r}26(2780) \\
120(1040) \\
790(450)\end{array}$ & & \\
\hline 614 & $\begin{array}{r}26(2770) \\
130(1190) \\
800(1390)\end{array}$ & $\begin{array}{c}28(2360) \\
180(760) \\
820(1060)\end{array}$ & \\
\hline 635 & $\begin{array}{r}25(1170) \\
160(1560) \\
800(1390)\end{array}$ & $\begin{array}{r}32(1150) \\
220(1350) \\
860(1190)\end{array}$ & $\begin{array}{l}140(890) \\
780(990)\end{array}$ \\
\hline 654 & $\begin{array}{l}200(910) \\
820(1060)\end{array}$ & $\begin{array}{l}230(1810) \\
910(1420)\end{array}$ & $\begin{array}{l}210(1620) \\
860(1840)\end{array}$ \\
\hline 668 & & & $\begin{array}{l}240(1630) \\
880(1700)\end{array}$ \\
\hline 695 & $\begin{array}{l}190(1880) \\
790(1890)\end{array}$ & $\begin{array}{l}220(1310) \\
890(1130)\end{array}$ & $\begin{array}{l}210(1530) \\
820(1520)\end{array}$ \\
\hline
\end{tabular}

times are found. These short-lived components could be due to either energy transfer or a fast photoinduced process like an isomerisation of a part of the excited chromophores. The latter process is likely because isolated subunits show, in contrast to integral PC, a low but distinct photoreversible absorption change [34]. Photochemistry may also be one cause for the comparably low degree of fluorescence polarization in the $\alpha$-subunit. A third component with a lifetime of typically around $200 \mathrm{ps}$ and low relative amplitude is detected for short wavelength excitation $(580 \mathrm{~nm})$, but not observed for 620 nm excitation.

It is not possible to decide at present whether these additional decay components are inherent also to "native" $\alpha$-subunit contained in integral PC, or whether they are due to heterogeneities present only in the isolated sub- 
unit. In view of the denaturation/renaturation sequence, and the time (approximately $4 \mathrm{~h}$ ) required for separation during which the subunits are unfolded, small deviations of the protein-chromophore arrangement from the "native" state can not be excluded. Theoretical estimates of the effects of such variations on the absorption and circular-dichroism spectra are given by Scharnagl et al. [35].

Despite these heterogeneities, the fluorescence decay of the $\alpha$-subunit is definitely dominated by the long-lived component, which contributes more than $95 \%$ of the total fluorescence. This is much improved over our previous preparations [18], and indicates that at least part of the heterogeneity observed earlier is a result of preparation artefacts. Based on these results, a lifetime of $1.1-1.2 \mathrm{~ns}$ can be assigned to those $\alpha-84$ chromophores which are embedded in the protein in a manner which compares with the native PC system (lifetime of fluorescing chromophores is about $1.2 \mathrm{~ns}$ ) [14 - 17].

\subsection{Fluorescence decay kinetics: $\beta$-subunit}

Figure 4 shows the time-resolved fluorescence of dimers of the $\beta$-subunit for $580 \mathrm{~nm}$ and $620 \mathrm{~nm}$ excitation and different detection wavelengths. The fit parameters of all measurements are summarized in Table 1. In Fig. 5, a plot of the analyzed lifetimes and amplitudes in dependence on the detection wavelength is given. Measurements were performed also with monomers prepared by detergent treatment (vide supra). The derived lifetimes and amplitudes were so close to those obtained for the dimers that only the results obtained with the much more stable dimers are discussed.

The $\beta$-subunit contains two chromophores between which energy transfer occurs via dipole-dipole interactions. Therefore, two exponentials should be necessary and sufficient to describe the time course of the fluorescence. One lifetime is related to the energy transfer and the second one to the lifetimes of the chromophores, if they are long compared with the energy transfer time (vide infra). The experimental decay curves, however, are characterized in most cases by a three-exponential decay. Only in some cases is a description with two exponentials sufficient (see Table 1).

For excitation with $580 \mathrm{~nm}$ and $600 \mathrm{~nm}$ light and for detection wavelengths up to $635 \mathrm{~nm}$, a component with a lifetime of $25-30 \mathrm{ps}$ is clearly apparent. For detection above $635 \mathrm{~nm}$, this short-lived decay is not observed, nor a corresponding rising component. The lifetime of the "intermediate" component varies between 120 and 240 ps with a tendency to increase with longer detection wavelengths. In all measurements this intermediate component occurs with significant amplitude. The third observed lifetime $(800-900 \mathrm{ps})$ is significantly shorter than the corresponding lifetime in the $\alpha$-subunit.

Since the chromophores of the $\beta$-subunit are connected by energy transfer, two decay rates $\left(k_{\mathrm{a}, \mathrm{b}}\right)$ are expected which are related to those of the individual chromophores $\left(k_{1}{ }^{\circ}\right.$ for $\beta-155, k_{2}{ }^{\circ}$ for $\left.\beta-84\right)$ and the 

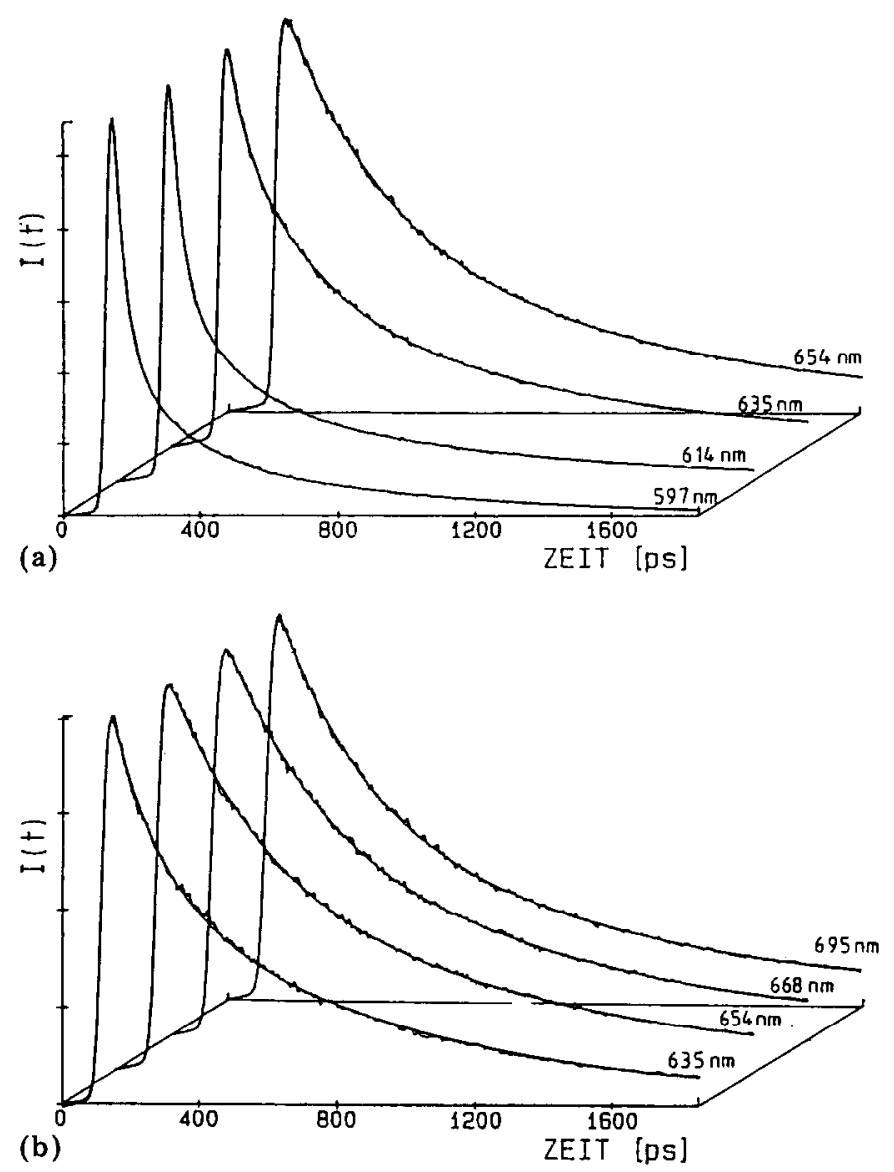

Fig. 4. Fluorescence decay of $\beta$-subunit (homodimer) in dependence on the detection wavelength. (a) $\lambda_{\text {exc }}=580 \mathrm{~nm}$; (b) $\lambda_{\text {exc }}=620 \mathrm{~nm}$.

rates of energy transfer ( $k_{12}$ and $k_{21}$ for transfer from and to $\beta-155$, respectively) via eqn. (1):

$k_{\mathrm{a}, \mathrm{b}}=\frac{1}{2}\left(k_{\mathrm{s}} \pm \sqrt{\left.k_{\mathrm{s}}{ }^{2}-4\left(k_{12} k_{2}^{\circ}+k_{21} k_{1}^{\circ}+k_{1}{ }^{\circ} k_{2}{ }^{\circ}\right)\right)}\right.$

where $k_{\mathrm{s}}=k_{1}^{\circ}+k_{2}^{\circ}+k_{12}+k_{21}$

When the theoretical values assigned to $k_{12}$ and $k_{21}$ are correct, then the inequity $k_{12}+k_{21} \gg k_{1}{ }^{\circ}+k_{2}{ }^{\circ}$ holds. In that case, eqn. (1) reduces to

$k_{\mathrm{a}}=k_{12}+k_{21}$

$k_{\mathrm{b}}=\frac{k_{12} k_{2}^{\circ}+k_{21} k_{1}^{\circ}}{k_{12}+k_{21}}$

Since the 25 ps component is mainly observed at short wavelength excitation, i.e. predominant excitation of the $\beta-155$ chromophore $[36,37]$, this 

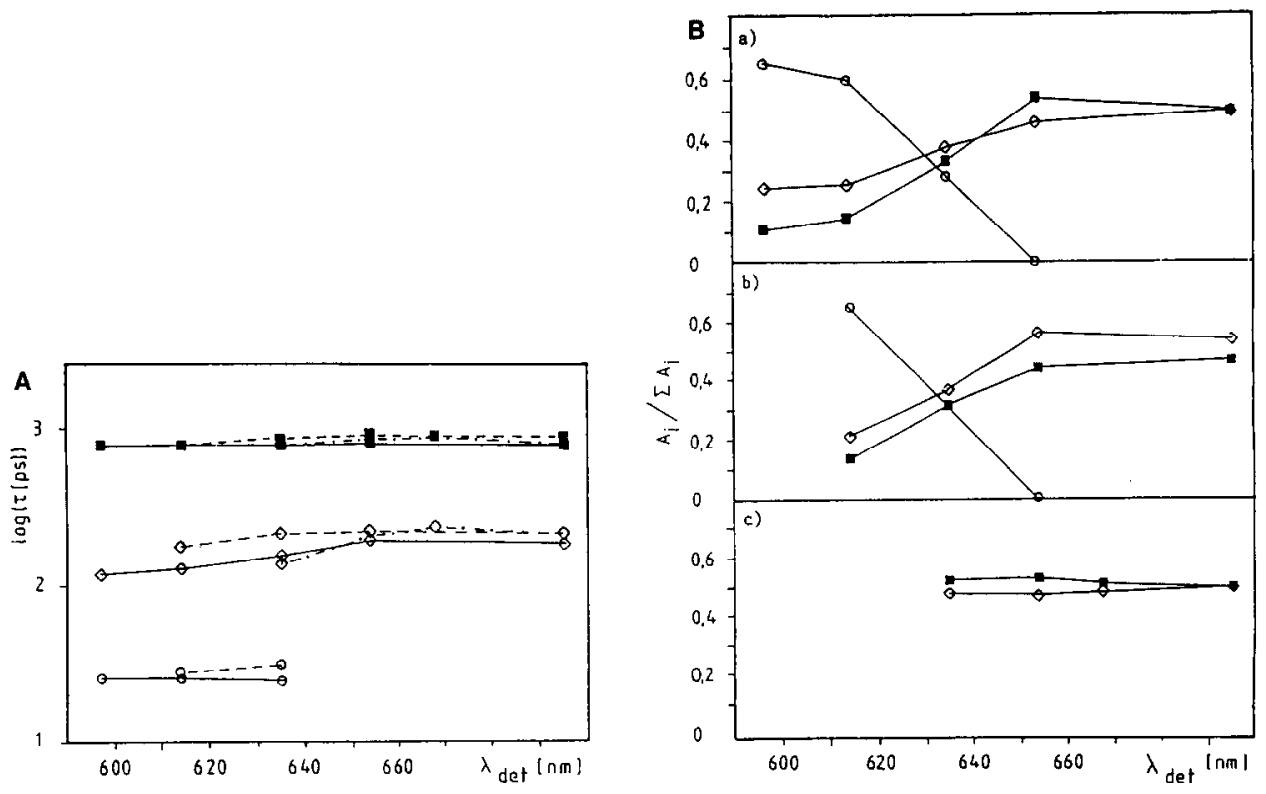

Fig. 5. Fluorescence decay fit parameters of the $\beta$-subunits. Dependence of components: fast $(O)$, medium $(\nabla)$, slow $(\nabla)$ on excitation and detection wavelengths. A) lifetimes with $\left.\lambda_{\text {exc }}=580 \mathrm{~nm}(-), \lambda_{\text {exc }}=600 \mathrm{~nm}(---), \lambda_{\text {exc }}=620 \mathrm{~nm}(-\cdot-) ; B\right)$ relative amplitudes with $\lambda_{\text {exc }}=580$ (a), 600 (b), $620 \mathrm{~nm}(\mathrm{c})$.

time constant can be assigned to the decay of this chromophore. According to eqn. (3a), the decay time is governed by the energy transfer rate to and from the $\beta-84$ chromophore $k_{12}$ and $k_{21}$ respectively. From theoretical calculations [13] it was concluded that both these transfer times are much shorter than the lifetimes of the individual chromophores $\left(k_{1}^{\circ}\right)^{-1}$ and $\left(k_{2}{ }^{\circ}\right)^{-1}$ respectively. It is then surprising that there is no fluorescence rise observed at long detection wavelengths, which would prove the indirect excitation of $\beta-84$ chromophores via energy transfer. A compensation occurs between the contributions of the fastdecay of "donor" fluorescence and the correspondingly rising "acceptor" fluorescence; this may be due to spectra overlap of the two chromophores, but other mechanisms cannot be ruled out.

Additional evidence for the interpretation of the fast decay time as energy transfer time is found in the difference curve $D(\mathrm{t})=I_{\|}(\mathrm{t})-I_{\perp}(\mathrm{t})$ (not shown). A fit produces a major component with a lifetime of about 30 ps. Since orientational relaxation of the protein to which this chromophore is attached is much slower, the fluorescence depolarization must be connected to energy transfer, or fast local motions. The latter is improbable in the native binding situation.

The lifetime of the third component $(800-900 \mathrm{ps})$ is considerably shorter than the longest lifetime in the $\alpha$-subunit. This is in accordance with the smaller steady-state fluorescence quantum yield of the $\beta$-subunit [38].

According to eqn. ( $3 \mathrm{~b}$ ) the longer one of the two lifetimes corresponds to a weighted average of the lifetimes of the two chromophores. Unless they 
have equal decay times (usually assumed in discussions on this topic), the lifetime of the chromophore with the shorter energy transfer time dominates in the evaluation of eqn. (3). Spectral studies have shown that the energy gap between the two chromophores is small enough that the back transfer ( $\beta-84$ to $\beta-155)$ is not negligible $[13,16,39-41]$. In this case, the observed decay time will be intermediate between the individual lifetimes of the two chromophores.

Two extreme cases result if one assigns a lifetime of $1.1-1.2 \mathrm{~ns}$ (e.g. that of the $\alpha$-chromophore) to either one of the two $\beta$-chromophores, and takes $k_{\mathrm{a}}=0.83-0.91 \mathrm{~ns}$ and $k_{\mathrm{b}}=37 \mathrm{ps}$ [13]. If $\beta-155$ is the longer-lived chromophore, then $\beta-84$ would have a lifetime of $790-810 \mathrm{ps}$; in the case of $\beta-84$ being the longer-lived one, then $\beta-155$ would have a lifetime of 400 . 450 ps. X-ray analysis has shown that the chromophore arrangements of $\beta-84$ and $\alpha-84$ are rather similar, and that both are close to the protein surface in monomers and subunits [9]. $\beta-155$ is much more twisted, and may be the one to become uncoupled first from the protein. It is then tempting to assume that its lifetime is significantly reduced compared with $\beta-84$ because conformational mobility is a cause for the rapid internal conversion in bile pigments $[5,42]$. However, $\beta-84$ seems to be involved in reversible photochemistry in partly denatured PC, and in conformational changes upon binding of linker polypeptides, which would favor this as the chromophore with a reduced lifetime. Clearly, more work is necessary to decide between the two possibilities.

More speculative is the assignment of the intermediate "200 ps" component. The relative amplitudes of this component are in general too high to be due to small fractions of deteriorated sample. Furthermore, the relative amplitude of this component shows a dependence on the detection wavelength, as does the amplitude of the long-lived component. This means that the emission spectrum of the species from which the 200 ps component originates corresponds fairly well to the $\beta-84$ chromophore. A possible explanation is that $\beta-84$ can adopt (at least) two different states (e.g. conformations, geometries or the like); one of them has a lifetime of about 800 $\mathrm{ps}$, the second one a lifetime of about $200 \mathrm{ps.} \mathrm{Since} \mathrm{the} \mathrm{relative} \mathrm{amplitudes}$ of both components increase with longer wavelengths of detection, the "second type" chromophore with the shorter lifetime must also act as an acceptor for energy being transferred from the $\beta-155$ chromophore.

The proposed explanation also seems to be reasonable on grounds of the information provided by X-ray analysis. The $\beta-84$ chromophore is not completely embedded by the surrounding protein. Therefore, the conformation of this chromophore is probably not as well fixed by the protein as it is for the $\alpha-84$ and the $\beta-155$ chromophores. The conformation of this chromophore may become more rigid in the intact antenna system because there it is fixed not only by the interaction with the $\alpha$-subunit, but also with the neighboring $\beta$-subunit and with linker polypeptides. The weaker stabilization of the $\beta-84$ chromophore results in different, nearly isoenergetic conformations in the isolated subunits with characteristically different lifetimes. 
Components with lifetimes of about 200 ps were also found in earlier measurements on chromopeptides. In these, the chromophores are not held by the protein in extended geometries as judged from their absorption and circular dichroism spectra [35]. In light of this explanation, the lifetime of $800-900$ ps must be assigned to the $\beta-84$ chromophore in a conformation which is close to the "native" one.

The complex decay kinetics are (at least in part) a manifestation of heterogeneities in the chromophore-protein arrangements of biliproteins. Their presence in the simplest form, e.g. the $\alpha$-subunit in its monomeric state, should be kept in mind when interpreting the fluorescence decay of higher aggregates with models based on perfect symmetry and/or identical arrangements of the individual chromophores (e.g. $\alpha-84, \beta-84, \beta-155$ in Cphycocyanin).

\section{Acknowledgments}

This work was supported by the Deutsche Forschungsgemeinschaft, 53 Bonn, F.R.G. (SFB 143, projects $\mathrm{A} 1$ and B1) and also by the Fonds der Chemischen Industrie. Mass culture of cells by Dr. G. Reng (Gesellschaft für Biotechnologische Forschung, 3301 Stöckheim, F.R.G.) is acknowledged. We also wish to thank Hamamatsu Photonics Europe for the loan of the synchroscan streak camera.

\section{References}

1 E. Gantt, Phycobilisomes. In L. A. Staehelin and C. J. Arntzen (eds.), Photosynthesis III, Springer, Berlin, 1986, pp. $260-268$.

2 D. A. Bryant, Phycobilisomes of Synechococcus sp. PCC 7002, Pseudoanabaena sp. PCC 7409 , and Cyanophora paradoxa: an analysis by molecular genetics. In H. Scheer and S. Schneider (eds.), Photosynthetic Light Harvesting Systems, W. De Gruyter, Berlin, 1988, pp. $217-233$.

3 A. N. Glazer, Light harvesting by phycobilisomes, Ann. Rev. Biophys: Chem., 14 (1985) $47-77$.

4 R. MacColl and D. Guard-Friar, Phycobiliproteins, CRC Press, Boca Raton, 1987.

5 H. Scheer, Light Reaction Path of Photosynthesis. In F. K. Fong (ed.), Phycobiliproteins: Molecular Aspects of Photosynthetic Antenna Systems, Springer, Berlin, 1982 , pp. $7-45$.

$6 \mathrm{~W}$. Wehrmeyer, Phycobiliproteins and phycobiliprotein organization in the photosynthetic apparatus of cyanobacteria, red algae, and cryptophytes. In U. Jensen and D. E. Fairbrother (eds.), Proteins and Nucleic Acids in Plant Systematics, SpringerVerlag, Berlin, 1983, pp. 144 - 167.

7 R. Rümbeli and H. Zuber, Isolation and characterization of components of the phycobilisomes from Mastigocladus laminosus and cross-linking experiments. In H. Scheer and S. Schneider (eds.), Photosynthetic Light Harvesting Systems, W. de Gruyter, Berlin, 1988, p. $61-71$.

8 D. J. Lundell, R. C. Williams and A. N. Glazer, Molecular architecture of a light harvesting antenna - in vitro. Assembly of the rod-substructures of synechococcus 6301 phycobilisomes, J. Biol. Chem., 256 (1981) 3580 - 3592. 
9 T. Schirmer, W. Bode and R. Huber, Refined three-dimensional structures of two cyanobacterial C-phycocyanins at 2.1 and $2.5 \mathrm{~A}$ resolution $-\mathrm{a}$ common principle of phycobilin-protein interaction, J. Mol. Biol., 196 (1987) 677 - 695.

$10 M$. Dürring and $R$. Huber, private communication (1989).

$11 \mathrm{H}$. Zuber, Primary structure and function of the light-harvesting polypeptides from cyanobacteria, red algae, and purple photosynthetic bacteria. In L. A. Staehelin and C. J. Arntzen (eds.), Photosynthesis III, Springer, Berlin, 1986, pp. 238 - 251.

12 D. A. Bryant, Genetic analysis of Phycobilisomes biosynthesis, assembly, structure, and function in the cyanobacterium Synechococcus sp. PCC 7002. In S. E. Stevens Jr. and D. A. Bryant (eds.), Light-energy Transduction in Photosynthesis: Higher Plant and Bacterial Models, American Society of Plant Physiologists, Rockville, MD, 1988, pp. 62 - 90.

$13 \mathrm{~K}$. Sauer and H. Scheer, Excitation transfer in C-phycocyanin. Förster transfer rate and excitation calculations based on new crystal structure data for C-phycocyanin from Agmenellum quadruplicatum and Mastigocladus laminosus, Biochim. Biophys. Acta, 936 (1988) 157 - 170.

14 A. Holzwarth, Picosecond fluorescence spectroscopy and energy transfer in photosynthetic antenna pigments. In J. Barber (ed.), Topics in Photosynthesis: The Light Reactions, Elsevier, 1987, pp. 95 - 157.

$15 \mathrm{H}$. Scheer, Excitation Transfer in Phycobiliproteins. In. L. A. Staehelin and C. J. Arntzen (eds.), Encyclopedia of Plant Physiology, Vol. 19, Photosynthesis III, Springer, Berlin, 1986, pp. 327 - 337.

16 M. Mimuro, P. Füglistaller, R. Rümbeli and H. Zuber, Functional assignment of chromophores and energy transfer in C-phycocyanin isolated from the thermophilic cyanobacterium Mastigocladus laminosus, Biochim. Biophys. Acta, 848 (1986) 155 166.

17 S. C. Switalsky and K. Sauer, Energy transfer among the chromophores of C-phycocyanin from Anabaena variabilis using steady-state and time-resolved fluorescence spectroscopy, Photochem. Photobiol., 40 (1984) $423-427$.

18 P. Hefferle, P. Geiselhardt, T. Mindl, S. Schneider, W. John and H. Scheer, Timeresolved polarized fluorescence of C-phycocyanin and its subunits from Mastigocladus laminosus, Z. Naturforsch., 39c (1984) 606-616.

19 A. Sandström, T. Gillbro, V. Sundström, R. Fischer and H. Scheer, Picosecond timeresolved energy transfer within C-phycocyanin aggregates of Mastigocladus laminosus, Biochim. Biophys. Acta 933 (1988) 42 - 53.

20 W. Köhler, J. Friedrich, R. Fischer and H. Scheer, Low temperature spectroscopy of cyanobacterial antenna pigments. In H. Scheer and S. Schneider (eds.), Photosynthetic Light Harvesting Systems, W. De Gruyter, Berlin, 1988, pp. 293 - 307.

21 W. Köhler, J. Friedrich, R. Fischer and H. Scheer, Site-selective spectroscopy and level ordering in C-phycocyanin, Chem. Phys. Lett., 143 (1988) $169 \cdot 173$.

22 S. Schneider, P. Geiselhart, F. Baumann, S. Siebzehnrübl, R. Fischer and H. Scheer, Energy transfer in "native" and chemically modified C-phycocyanin trimers and the constituent subunits. In $\mathrm{H}$. Scheer and S. Schneider (eds.), Photosynthetic Light Harvesting Systems. Organization and Function, Berlin, W. de Gruyter, 1988, pp. $469-483$.

23 A. N. Glazer, S. Fang and D. M. Brown, Spectroscopic properties of C-phycocyanin and its alpha- and beta-subunits, J. Biol. Chem., 248 (1973) $5679-5685$.

24 P. Füglistaller, H. Widmer, W. Sidler, G. Frank and H. Zuber, Isolation and characterization of phycoerythrocyanin and chromatic adaption of the thermophilic cyanobacterium Mastigocladus laminosus. Arch. Microbiol., 129 (1981) $268-274$.

25 E. Köst-Reyes, S. Schneider, W. John, R. Fischer, H. Scheer and H.-P. Köst, Fast preparative isoelectric-focusing of phycocyanin subunits in layers of granulated gels, Electrophoresis, 8 (1987), 335 - 336.

26 H. Scheer, Biliproteine, Angew. Chem., 93 (Angew. Chem. Int. Ed., 20241 - 261) (1981) $230-250$. 
27 R. W. Castenholz, Laboratory culture of thermophilic Cyanophytes, Schweizer Z. Hydrol., 35 (1970) 538 - 551.

$28 \mathrm{H}$. Scheer, W. Kufer, Conformational studies of Phycocyanin from Spirulina platensis, Z. Naturforsch. 32 c (1977) 513 - 518.

29 J. M. Burke, D. C. Pratt and A. Moskowitz, Low-temperature absorption and circular dichroism studies of Phytochrome, Biochemistry, 11 (1972), 4025 - 4031.

$30 \mathrm{H}$. Scheer, H. Formanek and S. Schneider, Theoretical studies of blliprotein chromophores and related bilepigments by molecular orbital and Ramachandran type calculation, Photochem. Photobiol., 36 (1982) $259-272$.

31 R. G. Martin, B. N. Ames, A method for determining the sedimentation behavior of enzymes: Application to protein mixtures, J. Biol. Chem., 236 (1961) $1372-1379$.

32 S. Siebzehnrübl. R. Fischer and H. Scheer, Chromophore Assignment in C-Phycocyanin from Mastigocladus laminosus, $Z$. Naturforsch., 42c (1987) 258 - 262.

33 P. Geiselhart, Dissertation, Technische Universität München, 1988.

34 G. Schmidt, S. Siebzehnrübl, R. Fischer and H. Scheer, Photochromic properties of C-Phycocyanin. In H. Scheer and S. Schneider (eds.), Photosynthetic Light Harvesting Systems. Organization and Function, W. de Gruyter, Berlin, 1988, pp. 77 - 89.

35 C. Scharnagl, Ph. D. Thesis, München, Technische Universität, 1988.

36 S. Siebzehnrübl, R. Fischer and H. Scheer, Chromophore Assignment in C-Phycocyanin from Mastigocladus laminosus, Z. Naturforsch., 42c (1987) 258 - 262.

37 T. Schirmer and M. G. Vincent, Polarized absorption and fluorescence spectra of single crystals of C-phycocyanin, Biochim. Biophys. Acta, 893 (1986) 379 - 385 .

38 R. Fischer, Diploma Thesis, Universität München, 1985.

39 F. W. J. Teale and R. E. Dale, Isolation and spectral characterisation of Phycobiliproteins, Biochem. J., 116 (1970) 161 - 169.

40 J. Grabowski and E. Gantt, Photophysical properties of Phycobiliproteins from Phycobilisomes: fluorescence lifetimes, quantum yields and polarization spectra, Photochem. Photobiol., 28 (1978) $39-45$.

41 M. Mimuro, R. Rümbeli, P. Füglistaller and H. Zuber, The microenvironment around the chromophores and its changes due to the association states in C-phycocyanin isolated from the cyanobacterium Mastigocladus laminosus, Biochim. Biophys. Acta, 851 (1986) 447 - 456.

42 W. Kufer, A. Holzwarth and H. Scheer, Isophorcarubin-A conformationally restricted and highly fluorescent bilirubin, Israel $J$. Chem., 23 (1983) 233 - 240. 